\title{
THE ESTIMATION OF THE INITIAL NUMBER OF BERTHS IN A PORT SYSTEM BASED ON COST FUNCTION
}

Wen-Chih Huang

Professor, Department of Harbor and River Engineering, National Taiwan Ocean University. Keelung, Taiwan 202, ROC, huangwc@mail.ntou.edu.tw

\section{Sheng-Chieh Wu}

Doctor Candidate, Department of Harbor and River Engineering, National Taiwan Ocean University. Keelung, Taiwan $202, R O C$

Follow this and additional works at: https://jmstt.ntou.edu.tw/journal

Part of the Civil and Environmental Engineering Commons

\section{Recommended Citation}

Huang, Wen-Chih and Wu, Sheng-Chieh (2005) "THE ESTIMATION OF THE INITIAL NUMBER OF BERTHS IN A PORT SYSTEM BASED ON COST FUNCTION," Journal of Marine Science and Technology. Vol. 13: Iss. 1, Article 5.

DOI: $10.51400 / 2709-6998.2102$

Available at: https://jmstt.ntou.edu.tw/journal/vol13/iss1/5

This Research Article is brought to you for free and open access by Journal of Marine Science and Technology. It has been accepted for inclusion in Journal of Marine Science and Technology by an authorized editor of Journal of Marine Science and Technology. 


\section{THE ESTIMATION OF THE INITIAL NUMBER OF BERTHS IN A PORT SYSTEM}

BASED ON COST FUNCTION

Acknowledgements

The authors would like to thank the National Science Council of the Republic of China for its support of the research NSC 89-2213-E-019-022. The content of this paper is part of the results from that study. 


\title{
THE ESTIMATION OF THE INITIAL NUMBER OF BERTHS IN A PORT SYSTEM BASED ON COST FUNCTION
}

\author{
Wen-Chih Huang* and Sheng-Chieh Wu**
}

Key words: cost function, the initial number of berths, queuing system, level of service (LOS).

\begin{abstract}
The process of optimizing a port queuing system, from a management perspective, is becoming more and more complicated. If we can find a way to measure the required minimum number and initial number of berths in a port system, and arrive at an optimal solution, we can save time. In this study, we have used many evaluation indexes, including cost functions, to estimate the initial number of berths for a port queuing system. According to our research results, we were able to estimate the initial number of berths $\left(\mathrm{N}_{0}\right)$ for a port queuing system by using the equation No $=\alpha+\beta \sqrt{\alpha}$, in which the relation between "traffic density $(\alpha)$ " and "the parameter of the initial facilities $(\beta)$ " in the system, changes with the different evaluation indexes and levels of service.
\end{abstract}

\section{INTRODUCTION}

The planning of public service facilities consists of three tasks: demand forecasting, capacity analysis, and the determination of optimal number of service facilities. In this study, we have used a port system as an example, to discuss the relation between the number of facilities and the evaluation indexes used to measure the system's demand and supply. Generally speaking, while applying queuing theory to calculate the optimal number of facilities within a system, the average service rate $(\mu)$ of the system's facility is often considered as a constant, as in this way, a solution can be more easily obtained. Looking at this problem from the perspecive of management, it seems like the construction models, and factors which must be considered, have become more and more complicated. The factors affecting a

Paper Submitted 12/08/03, Accepted 11/04/04. Author for Correspondence: Wen-Chih Huang. E-mail: huangwc@mail.ntou.edu.tw.

*Professor, Department of Harbor and River Engineering, National Taiwan Ocean University. Keelung, Taiwan 202, ROC.

**Doctor Candidate, Department of Harbor and River Engineering, National Taiwan Ocean University. Keelung, Taiwan 202, ROC. service facility include operating equipment, operating efficiency, the interaction of operating equipment, the peak characteristics of the operation system and the factor cause by dwell time. Therefore, in consideration of these complicated changing conditions, we have used a system simulation to seek a solution to determine the optimal number of facilities and service rate. It is crucial that the initial number of facilities in the system be determined correctly. In previous system simulations, by inputting the initial number of facilities from the very beginning, much time has been wasted. If the required minimum number of port system service facilities can be measured, we can shorten the time required to reach a solution for the optimal number of berths.

In this study, two types of system evaluation indexes have been considered. The first being queuing indexes, including Degree of Congestion (DC), Average waiting time $\left(W_{q}\right)$, and Waiting time factor $\left(W_{q} \cdot \mu\right)$; the second evaluation factor being the cost function. Use of these indexes can assist us in estimating the initial number of berths for a port queuing system. Based on all the problems outlined in prior studies, we have provided a convenient way (by looking up diagrams or Tables) to derive the minimum number of service facilities in a queuing system, reaching the optimal solution according to expected traffic density and level of service. This offers a timely solution in a situation where the initial number of facilities exceeds the optimal number of facilities.

The remainder of this paper is organized as follows. Part two illustrates the basic principles of the queuing system. Part three defines the types of evaluation indexes for a port system. Part four introduces and compares several methods of determining the initial number of facilities in a port queuing system. Part five gives some examples to illustrate the applicable range and effectiveness of our research method, while part six describes our empirical results. Finally, we present our conclusion . 


\section{PORT QUEUING SYSTEM}

Ports generally use a classical type of queuing system. Previous related studies on the optimal number of berths for a port's queuing system include: Plumlee [8], Nicolau [5], Wanhill [9], Noritake [6], Noritake and Kimura [7] and Huang [1]. This study focuses on the $M /$ $M / N$ queuing model: the first $M$ signifies that customers' arrival intervals follow an Exponential distribution; the second $M$ signifies that service time follows an Exponential distribution; while the $N$ represents the number of service facilities.

The $M / M / N$ model is explained below: The $M / M / N$ model is representative of a system in which there are $N$ $(N>1)$ numbers of the same serving facilities, because the system's capacity is unlimited with the arrival rate of $\lambda$.

$$
\lambda_{n}=\lambda \quad \forall n \geq 0
$$

Concerning service time, because the system consists of $N$ serving facilities, the largest number of customers that can receive service is $N$. Meanwhile, when the number of customers is less than $N$, there will be $\mathrm{n}$ number of customers receiving service, and $N-n$ number of service facilities left unused. Thus we can write

$$
\mu_{n}= \begin{cases}n \mu, & \text { if } n=1,2, \ldots, N-1 \\ N \mu, & \text { if } n=N, N+1, \ldots\end{cases}
$$

Let $\lambda_{n}, \mu_{n}$ into $\prod_{i=1}^{n} \frac{\lambda_{i-1}}{\mu_{i}}$, we have

$$
\prod_{i=1}^{n} \frac{\lambda_{i-1}}{\mu_{i}}= \begin{cases}\frac{\lambda^{n}}{n ! \mu^{n}}, & \text { if } n=1,2, \ldots, N-1 \\ \frac{\lambda^{n}}{N^{n-N} N ! \mu^{n}}, & \text { if } n=N, N+1, \ldots\end{cases}
$$

Using (1) and (3), the possibility a $P_{0}$ being a balanced equation is as follows.

$$
\begin{aligned}
p_{0} & =\left[1+\sum_{n=1}^{N j-1} \frac{\left(\frac{\lambda}{\mu}\right)^{n}}{n !}+\frac{\left(\frac{\lambda}{\mu}\right)^{N}}{N !} \sum_{n=N}^{\infty}\left(\frac{\lambda}{N \mu}\right)^{n-N}\right]^{-1} \\
& =\left[\sum_{n=0}^{N-1} \frac{\left(\frac{\lambda}{\mu}\right)^{n}}{n !}+\frac{\left(\frac{\lambda}{\mu}\right)^{N}}{N !} \frac{1}{1-\frac{\lambda}{N \mu}}\right]^{-1}
\end{aligned}
$$

Judging from the above two equations, the condition for an existing solution in a steady state is $\rho=\lambda /(N$ $\cdot \mu)<1$. Therefore, in seeking the optimal number of system facilities, we can regard this condition as the basic condition for seeking the optimal number of system facilities. Moreover, when applying this condition to the equation, we can see that.

$$
p_{n}= \begin{cases}\frac{\lambda^{n}}{n ! \mu^{n}} p_{0}, \quad \text { if } n=1,2, \ldots, N-1 \\ \frac{\lambda^{n}}{N^{n-N} N ! \mu^{n}}, \text { if } n=N, N+1, \ldots\end{cases}
$$

\section{EVALUATION INDEXES FOR A PORT SYSTEM}

Plumlee [8] adopted a distribution of ship arrivals at ports, with the relation between the average number of ship arrivals and berth numbers. This paper considered the relation between berth usage at the lowest total cost of idle berths, ship waiting costs and number of berths. Nicolau [5] concluded that planning for a port facility must be in accordance with the requirements of ship companies and port operators. The ideal situation is that all berths are occupied during the time available, and no ship is ever kept waiting. Wanhill [9] indicated that in evaluating the cost function of the optimal number of port system facilities, besides considering the cost of the idle berths and the cost of ships' waiting time, it must also include a ship's costs during service time in port. Noritake [6] and Noritake and Kimura [7] thought that determining the number of public wharf berths should reflect variations in the state of cargo demand. In addition, they took the costs of both berths and ships into account. Thus, the calculation can be completed by means of the cost ratio between berths and ships, and the average number of ships in port. Huang [1] developed a evaluation index (IND) for a port, which was mediated on several kinds of cost functions (including ships' costs and interest on cargo, as well as equipment, construction and maintenance costs, management costs of ports' facilities, the cost of installing and removing the machinery and the cost of its operators, costs of storing cargo and so on). By using these indexes, we can analyze a port systems' characteristics at different time periods and in various ports.

Utilizing the aforementioned paper's evaluation indexes can serve to measure a port system's efficiency. This study has divided these evaluation indexes into two types, based on features of queuing theory and cost functions. The definition of these indexes is laid out below.

\section{Evaluation indexes related to queuing systems}

\section{(1) Degree of congestion (DC)}

$D C$ is the probability of having to wait for a berth 
when a vessel arrives in port.

$$
D C=\sum_{j=N+1}^{\infty} P_{N}(j)
$$

Where, $N$ is the number of berths, and $P_{N}(j)$ is the probability of $j$ vessels arriving in the port within the period $T$

\section{(2) Utilization $(\rho)$}

$\rho$ is the ratio of actual berth utilization time to the gross berth time available.

$$
\begin{aligned}
& \rho=1-\left(\sum_{j=0}^{N-1}(N-j) P_{N}(j) / N\right) \\
& =\left(\sum_{j=1}^{N} j \cdot P_{N}(j) / N\right)+D C
\end{aligned}
$$

\section{(3) Average number of ships in port ( $L)$}

$L$ is the expected number of ships arriving in the port within the period $T$.

$$
L=\sum_{j=0}^{\infty} j \cdot P_{N}(j)
$$

(4) Average number of ships in queue $\left(L_{q}\right)$

$L_{q}$ is the expected number of ships waiting for service in the port system.

$$
L_{q}=\sum_{j=N+1}^{\infty}(j-N) \cdot P_{N}(j)
$$

(5) Average waiting time (W) in the port system.

$$
W=L / \lambda
$$

Where, $\lambda$ is the mean arrival rate of vessel.

(6) Average waiting time $\left(W_{q} ; A W T\right)$

$W_{q}$ is the mean time a vessel spends waiting in line for service.

$$
W_{q}=L_{q} / \lambda
$$

(7) Waiting time factor $\left(W_{q} \cdot \mu\right.$; AWT/AST)

$W_{q} \cdot \mu$ is the ratio of the average waiting time $\left(A W T ; W_{q}\right)$ to average service time $(A S T ; 1 / \mu)$.

$$
W_{q} \cdot \mu=A W T / A S T
$$

According to the definitions of these evaluation indexes, the system's steady state probability of the $M /$
$M / N$ queuing system can be expressed as:

$$
P_{N}(j)= \begin{cases}\frac{\left(\frac{\lambda}{\mu}\right)^{j} \cdot P_{N}(0)}{j !} & (0 \leq j<N) \\ \frac{P_{N}(0)}{N ! \cdot N^{(j-N)}} & (j \geq N)\end{cases}
$$

Where,

$$
P_{N}(0)=\frac{1}{\left[\sum_{j=0}^{\infty} \frac{\left(\frac{\lambda}{\mu}\right)^{j}}{j !}+\frac{\left(\frac{\lambda}{\mu}\right)^{N}}{N !(1-\rho)}\right]}
$$

Using (14) into (6) (13) we show:

$$
\begin{aligned}
& D C=1-\sum_{j=0}^{N} \frac{P_{N}(0) \cdot\left(\frac{\lambda}{\mu}\right)^{j}}{j !} \\
& \rho=1-\sum_{j=0}^{N-1}\left[\frac{(N-j) \cdot P_{N}(0) \cdot\left(\frac{\lambda}{\mu}\right)^{j}}{j ! \cdot N} \mid\right. \\
& L_{q}=\frac{P_{N}(0) \cdot\left(\frac{\lambda}{\mu}\right)^{j} \cdot \rho}{j ! \cdot(1-\rho)^{2}} \\
& W_{q}=\frac{L_{q}}{\lambda}=\frac{P_{N}(0) \cdot\left(\frac{\lambda}{\mu}\right)^{j} \cdot \rho}{j ! \cdot(1-\rho)^{2} \cdot \lambda} \\
& W_{q} \cdot \mu=\frac{P_{N}(0) \cdot\left(\frac{\lambda}{\mu}\right)^{j}}{j ! \cdot(1-\rho)^{2} \cdot N}
\end{aligned}
$$

\section{Evaluation indexes related to cost function}

Decisions related to cost functions in port planning are key factors in optimizing the facilities. Accordingly, in this section, these decisions are looked at in light of a cost function's type and definition. By looking at the years since 1960, the cost functions of port systems, presented in prior studies, can be categorized into three major types. Plumlee [8] employs the minimizing of a ship's waiting cost, and a berth's idle cost, to present the first type of cost function $T C_{1}$.

$$
T C_{1}=U_{s} \cdot \lambda \cdot W_{q}+U_{b} \cdot N(1-\rho)
$$


Where $U_{s}$ is the cost of a ship per unit of time; $U_{b}$ is the cost of a berth per unit of time. Nicolau [5] only considered the cost of waiting berths, as well as the idle cost and operation cost of berths, thus forming $T C_{2}$, defined as follows:

$$
T C_{2}=U_{s} \cdot \lambda \cdot W_{q}+U_{b} \cdot N
$$

Where $U_{b} \bullet N$ are the cost of berths, including berths' idle cost and operation cost.

Wanhill [9] and Noritake and Kimura [7] added the cost of service time for ships staying in port to $T C_{2}$, thus forming the widely used cost function $T C_{3}$.

$$
T C_{3}=U_{s} \cdot \lambda \cdot\left(W_{q}+1 / \mu\right)+U_{b} \cdot N
$$

Most papers have combined the indexes of the queuing system and the total cost function to create major indexes to measure port systems, thus creating a standard to measure the optimal port system. The definitions of the cost indexes, referred to in these papers, vary. Nicolau [5] defined the cost function as: ships' waiting cost divided by berths' idle cost plus ships' waiting cost $\left(C_{s} /\left(C_{b}+C_{s}\right)\right.$. This ratio can be used as an index to judge optimal port cost, the degree of congestion and berth utility. Noritake and Kimura [7] used the ratio between berths' cost and ships' $\operatorname{cost}\left(\gamma_{b s}\right.$ $\left.=C_{b} / C_{s}\right)$ as the basis for deciding the optimum number of berths in a public wharf. Huang [1], and Huang et al. [2], defined the total cost of a ship in port as the cost of ship and cargo $\left(C_{1}\right)$ and the terminal service cost $\left(C_{2}\right)$. $C_{1}$ and $C_{2}$ are defined as

$$
C_{1}=C_{s}+C_{c g}
$$

Where, $C_{s}$ is the ship's cost, including construction, maintenance and operation expenditures, and $C_{c g}$ is the cargo loaded aboard, and the interest cost of related equipment.

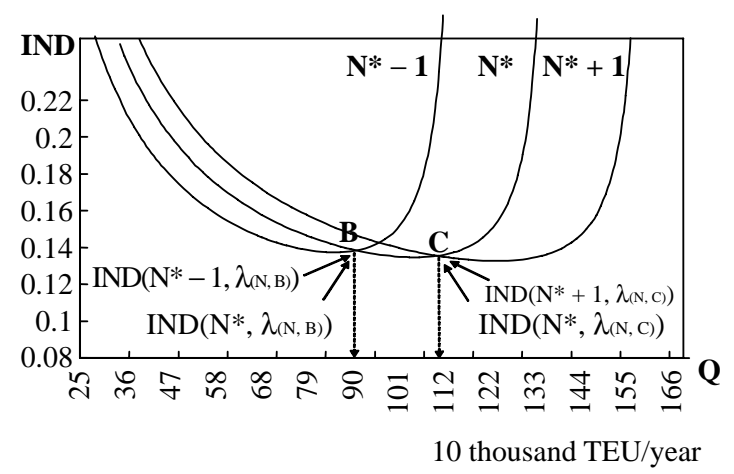

Fig. 1. Optimal berths capacity in a port system with IND.

$$
C_{2}=C_{p f}+C_{p o}+C_{b f}+C_{b o}+C_{c m}+C_{c o}+C_{y d}
$$

Where,

$C_{p f}, C_{p o}$ : the construction of port facilities and their operation costs.

$C_{b f}, C_{b o}$ : pier construction and operation costs,

$C_{c m}$ : machinery handling and maintenance costs,

$C_{c o}$ : working costs of machinery operators,

$C_{y d}$ : storage yard costs.

The total cost is then obtained, as follows:

$$
\begin{aligned}
T C & =C_{1}+C_{2} \\
& =\left(U_{s}+U_{c g} \cdot X\right)\left(\lambda / \mu+L_{q}\right)+\left(U_{p f}+U_{p o}+U_{b f}\right. \\
& \left.+U_{b o}\right) \cdot N+U_{c m} \cdot N \cdot A C+U_{c o} \cdot A C \cdot T \cdot \lambda \\
& +U_{y d} \cdot V \cdot H \cdot \lambda
\end{aligned}
$$

Where, $V$ is the average cargo volume of a ship in port and $H$ is the average relay time of each cargo.

Huang [1] and Huang et al. [2] used a index (IND) to estimate the optimal number of berths in a port system; IND is defined as:

$$
\begin{aligned}
\mathrm{IND} & =\mathrm{TC} /\left(\lambda \cdot U_{s} \cdot V\right)=\left(\left(1+R_{c g}\right)\left(1 / \mu+W_{q}\right)\right. \\
& +\left(R_{p f}+R_{p o}+R_{b f}+R_{b o}\right) \cdot N / \lambda+R_{c m} \cdot N \\
& \left.\cdot \mathrm{AC} / \lambda+R_{c o} \cdot \mathrm{AC} \cdot T+R_{y d} \cdot V \cdot H\right) / V
\end{aligned}
$$

Where, $R_{c g}, R_{p f}, R_{p o}, R_{b f}, R_{b o}, R_{c m}, R_{c o}$, and $R_{y d}$ are the cost ratios of $U_{c g} \cdot \mathrm{X}, U_{p f}, U_{p o}, U_{b f}, U_{b o}, U_{c m}, U_{c o}, U_{y d}$ divided by $U_{s}$ respectively.

Figure 1 shows the range of the optimal capacity in a port, with IND at the lowest range. If the number of berths is $N^{*}$, it must satisfy the Eq. (28ab).

$$
\begin{aligned}
& \operatorname{IND}\left(N^{*}-1, \mu_{(N, B)}\right) \geq \operatorname{IND}\left(N^{*}, \lambda_{(N, B)}\right) \text { and } \\
& \operatorname{IND}\left(N^{*}+1, \lambda_{(N, C)}\right) \geq \operatorname{IND}\left(N^{*}, \lambda_{(N, C)}\right)
\end{aligned}
$$

From (27) into (28a), we have

$$
\begin{aligned}
& {\left[( 1 + R _ { c g } ) \left(1 / \mu+W_{q}\left(N^{*}-1, \lambda_{(N, B)}\right)\right.\right.} \\
& +\left(R_{p}+R_{b}\right)\left(N^{*}-1\right) / \lambda_{(N, B)}+R_{c m}\left(N^{*}-1\right) \\
& \left.\left.\cdot \mathrm{AC} / \lambda_{(N, B)}+R_{c o} \cdot T \cdot \mathrm{AC}+R_{y d} \cdot H \cdot V\right)\right] / V \\
& \geq\left[( 1 + R c g ) \left(1 / \mu+W_{q}\left(N^{*}, \lambda_{(N, B)}\right)\right.\right. \\
& +\left(R_{p}+R_{b}\right)\left(N^{*}\right) / \lambda_{(N, B)}+R_{c m}\left(N^{*}\right) A C / \lambda_{(N, B)}
\end{aligned}
$$


$\left.\left.+R_{c o} \cdot T \cdot A C+R_{y d} \cdot H \cdot V\right)\right] / V$

Then,

$$
\begin{aligned}
& \lambda_{(N, B)}\left(W_{q}\left(N^{*}-1, \lambda_{(N, B)}\right)-W_{q}\left(N^{*}, \lambda_{(N, B)}\right)\right. \\
& \geq\left(R_{p}+R_{b}+R_{c m} \cdot A C\right) /\left(1+R_{c g}\right)
\end{aligned}
$$

Because

$$
\begin{aligned}
& \lambda \cdot W_{q}=L_{q} \\
& L_{q}\left(N^{*}-1, \lambda_{(N, B)}\right)-L_{q}\left(N^{*}, \lambda_{(N, B)}\right) \\
& \geq\left(R_{p}+R_{b}+R_{c m} \cdot A C\right) /\left(1+R_{c g}\right)
\end{aligned}
$$

in the same way from (27) into (28b), we have

$$
\begin{aligned}
& L_{q}\left(N^{*}+1, \lambda_{(N, C)}\right)-L_{q}\left(N^{*}, \lambda_{(N, C)}\right) \\
& \geq\left(R_{p}+R_{b}+R_{c m} \cdot A C\right) /\left(1+R_{c g}\right)
\end{aligned}
$$

According to $(31,32)$, we have

$$
\begin{aligned}
& L_{q}\left(N^{*}, \lambda_{(N, C)}\right)-L_{q}\left(N^{*}+1, \lambda_{(N, C)}\right) \\
& \leq\left(R_{p}+R_{b}+R_{c m} \cdot A C\right) /\left(1+R_{c g}\right) \\
& \leq L_{q}\left(N^{*}-1, \lambda_{(N, B)}\right)-L_{q}\left(N^{*}, \lambda_{(N, B)}\right)
\end{aligned}
$$

Therefore,

$$
\begin{aligned}
& L_{q}\left(N^{*}, N^{*}+1, \lambda_{(N, C)}\right) \leq \mathrm{CI} \leq L_{q}\left(N^{*}-1, N^{*}, \lambda_{(N, B)}\right) \\
& \mathrm{CI}=\left(U_{p}+U_{b}+U_{c m} \cdot A C\right) /\left(U_{s}+U_{c g}\right) \\
& \quad=\left(R_{p}+R_{b}+R_{c m} \cdot A C\right) /\left(1+R_{c g}\right)
\end{aligned}
$$

Where, $U_{p}$ is the unit time cost of the port; $U_{c m}$ is the unit time cost of the cranes; $A C$ is the number of cranes; the unit time cost of cargo on board and relevant equipment is given by $U_{c g}$, and $R_{p}, R_{b}, R_{c m}$, $R_{c g}$, are the ratios between the aforesaid cost items and $U_{s}$ (the average waiting cost per ship), respectively. Because this cost index considers the more complete cost items of the port system, CI was adopted as the evaluation indexes of the cost function for this study.

Combining the above analyses, this study has considered four evaluation indexes, including Degree of Congestion (DC), Average of waiting time $\left(W_{q}\right)$, Waiting time factor $\left(W_{q} \cdot \mu\right)$ and Cost Index (CI) as the evaluation indexes to research port system facility planning.

\section{THE DETERMINITION OF THE INITIAL NUMBER OF BERTHS}

This study has incorporated past research into the initial number of facilities for an optimal queuing system; the calculation method is laid out below:

\section{The minimum number of service facilities in queuing system}

Plumlee [8], Nicolau [5] and Noritake [6], used the utilization of berths to deduce the condition of the minimum number of berths in the $M / M / N$ queuing system. An explanation of the model and notations are given below:

$N$ : Number of berths

$T$ : Period of time considered for port operation, usually 1 year $=365$ days .

$Q$ : Total tonnage of general cargo loaded onto and discharged from ships in port during the period $T$.

$R$ : Daily rate of cargo handling per berth.

$N_{p}$ : Average number of ships present in port with $T$ berths during the period $T$.

$N_{b}$ : Average number of ships served by $T$ berths during the period $T$.

$N_{w}$ : Average number of ships waiting at port with $T$ berths during the period $T$.

$P_{N}(j)$ :Probability of $\mathrm{j}$ ships being present in port with $T$ berths during the period $T$.

$F_{N}(j)$ :Average day having $\mathrm{j}$ ships present in port with $T$ berths during the period $T$.

Where $\sum_{j=0}^{\infty} P_{N}(j)=1$

$$
\begin{gathered}
F_{N}(j)=T \cdot P_{N}(j) \\
\sum_{j=0}^{\infty} F_{N}(j)=T
\end{gathered}
$$

The degree of occupation of $\mathrm{N}$ berths in a port was referred to as the "degree of occupancy" by Nicolau [5]. For example,

$$
\begin{aligned}
& \rho=\frac{\sum_{n=1}^{N} j \cdot F_{N}(j)+N \cdot \sum_{j=N+1}^{\infty} F_{N}(j)}{N \cdot \sum_{j=0}^{\infty} F_{N}(j)} \\
& \left.=\frac{\sum_{j=0}^{N} j \cdot P_{N}(j)+N \cdot \sum_{j=N+1}^{\infty} P_{N}(j)}{N}\right]<1
\end{aligned}
$$


$N_{b}=$ average number of ships served at a port with $N$ berths during the period $T$; Noritake and Kimura [7] defined the relation parameters as follows:

$$
\begin{aligned}
& N_{b}=\sum_{j=0}^{N} j \cdot P_{N}(j)+N \cdot \sum_{j=N+1}^{\infty} P_{N}(j)=N \cdot \rho \\
& N_{w}=\sum_{j=N+1}^{N} j \cdot P_{N}(j)+N \cdot \sum_{j=N+1}^{N} P_{N}(j) \\
& N_{p}=\sum_{j=0}^{N} j \cdot P_{N}(j)=N_{b}+N_{w} \\
& Q=R T\left(\sum_{j=0}^{N} j \cdot P_{N}(j)+N \cdot \sum_{j=N+1}^{\infty} P_{N}(j)\right)
\end{aligned}
$$

From (40), (41)

$$
Q=N \cdot R \cdot T \cdot \rho=N_{p} \cdot R T
$$

Then,

$$
\frac{Q}{R T}=N \cdot \rho=N_{p}
$$

Accordingly, (40) $\rho<1$ and using (46), we have

$$
N>\frac{Q}{R T}
$$

Equation (47) shows the condition for the required minimum number of berths to deal with cargo quantity $Q$ in the port queuing system.

\section{Normal distribution estimating number of customers}

Newell [4] used a normal distribution approximation to estimate the number of customers, by presenting a decision method for calculating the initial number of service facilities. When the parameters are very large, the Poisson distribution will approximate a normal distribution; its average number and the parameter number both being $(\lambda / \mu)$. Therefore, when $1<N<\infty$, the probability of the equation can be approximated by the following equation.

$$
P_{n} \approx A \exp \left[\frac{-\left(n-\frac{\lambda}{\mu}\right)^{2}}{2\left(\frac{\lambda}{\mu}\right)}\right]
$$

Where, $A=\sqrt{(2 \pi \lambda / \mu)}$

When drawing the diagram, according to this equation, we find at the geometric distribution of $n>N$, that no matter what the sliding rate or lineage shape of the curve may be, it can meet a normal distribution, as shown in Figure 2, at the place where $\mathrm{n}$ is equal to $N$. In Figure 2, the points joined by solid lines represent the probability pn for $n$ customers in an $\mathrm{m}$-channel server with $\lambda / \mu$ $=16$; the points joined by a broken line represent approximate values obtained by joining a normal distribution to an exponential distribution. Accordingly, the whole $M / M / N$ system can employ a normal distribution to estimate the value of $N$.

Lan and Teng [3] applied the concept of Newell [4] to analyze the transient behavior of the $M / M / N$ queuing system, and presented a simple method of designing the number of service facilities. That is, they used the characteristic of probability Pn for the state of the queuing system, using traffic density, plus one or two times the standard deviation, to decide the minimum required number of service berths for the port system. However, this method doesn't consider which evaluation indexes can be applied to the system's level of service. Thus, for practical application, if we use $[(\lambda / \mu)$ $+(1 \sim 2) \sqrt{(\lambda / \mu)}]$ as the initial number, the solution is frequently larger than the optimal number of facilities. Therefore, it is necessary to seek a more correct method to decide the initial number of system facilities.

\section{Method of determining the initial number of system facilities}

After looking at various kinds of decision methods for the optimal number of service facilities, this study used the concept of traffic density, plus one or two times the standard deviation, to decide the initial number of facilities. In addition, in light of the disadvantages of the aforementioned studies, we considered the following factors for revision:

1. How the cost function's indexes influence the system's initial number of facilities (for example, CI).

2. How different systems' evaluation indexes influence the initial number of facilities.

3. How different traffic densities influence the initial number of facilities.

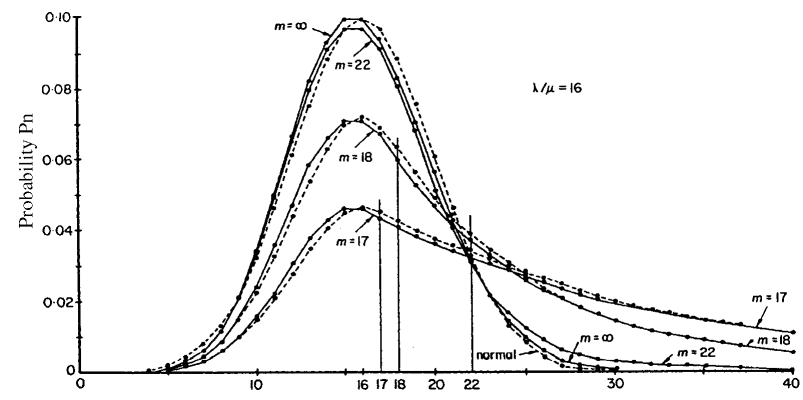

Fig. 2. The number of customers (n) in the system [4]. 
4. How the different characteristics of the initial number of facilities are influenced by diverse levels of service.

This study, therefore, considered how different kinds of evaluation indexes, under diverse traffic densities and levels of service, would influence the deciding number of initial facilities in a system. Newell's [4] method can be written as follows:

$$
N_{o}=\frac{\lambda}{\mu}+(1 \sim 2) \sqrt{\frac{\lambda}{\mu}}
$$

The solution for the initial number of facilities, in equation (49), in given for the worst level of service, which is probably larger than the optimal number of facilities, because this method is only partially applicable. This study revised the part of equation (49), which uses one or two times the initial number's parameter $(\beta)$, and demanded the traffic density to be $\alpha=\lambda / \mu$. The revision is shown as equation (50).

$$
N_{o}=\alpha+\beta \sqrt{\alpha}
$$

\section{MEASURING THE PARAMETERS OF INITIAL NUMBER OF FACILITIES}

\section{Range of indexes under different levels of service}

According to the above analysis, the initial number of facilities varies, more or less, in accordance with the kinds of evaluation indexes, and is associated with the levels of service within these evaluation indexes. Therefore, based on these different indexes, this study divided their levels of service into levels A, B and C. Taking the degree of congestion as an example, the range of level $\mathrm{A}$ was between $0 \%$ to $20 \%$; that is, the probability of having to wait, because of system congestion, was only about $0 \%$ to $20 \%$. The range for level B was about $20 \%$ to $50 \%$, while for level C it was $50 \%$ to $70 \%$. Of course, the DC would be greater than $70 \%$, if $\mathrm{DC}>70 \%$, then $\beta \rightarrow 0, N_{o}=\alpha$. As a matter of course, level A offered a higher level of service. The classifications of other evaluation indexes are shown in Table 1.

Table 1. Range of port indexes under different to levels of service

\begin{tabular}{cccc}
\hline L. O. S. & Level A & Level B & Level C \\
indexes & & & \\
\hline DC & $0-20 \%$ & $20 \%-50 \%$ & $50 \%-70 \%$ \\
$W_{q}$ & $0-3 \mathrm{hrs}$ & $3-6 \mathrm{hrs}$ & $6-12 \mathrm{hrs}$ \\
$W_{q} \cdot \mu$ & $0-0.15$ & $0.15-0.3$ & $0.3-0.5$ \\
$\mathrm{CI}$ & $0-0.5$ & $0.5-1.0$ & $1.0-5.0$ \\
\hline
\end{tabular}

\section{Measuring parameters for the initial number of facilities}

For the benefit of comparison and analysis, this section uses the $\mathrm{M} / \mathrm{M} / \mathrm{N}$ model to explain how the initial number of facilities was determined. In order to determine the system's initial number of facilities, we used the optimal number of facilities from the above indexes, under diverse traffic densities and levels of service.

Taking the cost index (CI) as an example, when the traffic density $\alpha=1.0$, the optimal number of facilities should be 3 , in order to maintain its service level of CI $=0.3$. Based on Newell [4] and Lan and Teng [3], by using the initial number of the standard deviation at 1 to 2 times its traffic density $(\lambda / \mu)$, the value of 2 is the optimal number of berths when the standard deviation being of 1 time is planted into the initial solution of the simulation. Had twice the standard deviation been employed to resolve the initial number of berths, the optimal solution would be the initial simulation solution, which was 3 . When $\alpha=4.0, \mathrm{CI}=0.3$ the optimal number of facilities would be 7 , with the initial solution for the other method being 6 and 8 . With an initial solution for the simulation of 8 , this is well above the optimal solution. It is understood that the resolution for the initial number of facilities for the $M / M / N$ queuing system, put forward in the literature by Newell [4] and Lan and Teng [3], is a local solution only, as marked in Figure 3. In order to solve the problem, this study determined the smallest number of facilities within each range and the value of this relationship to traffic density. This is referred to, in this study, as "the parameter of initial facilities $(\beta)$ ". Based on these relationship values, the relationship between traffic density and the initial parameter value of the facilities can be mapped.

As seen in Figure 3, it is represented by three curves of initial numbers of facilities, under three different service levels; when $\alpha=1.0, \mathrm{CI}=0.3$. According to Figure 3 and equation (50), the value of the initial number's parameter $(\beta)$ is 1.3 , and the initial number of berths (No) is 2.3. When $\alpha=2.0, \mathrm{CI}=0.3$, then obtaining $\alpha=$ 1.2, No $=3.7$. Furthermore, such curves stand for the relationship between traffic density and the initial parameter value of the facilities. These can be formulated as equations (51) to (53) under different levels of service:

$$
\begin{aligned}
& \text { A level of service }(\mathrm{CI}=0 \sim 0.5) \\
& \beta=1.1+2.8 \cdot e^{-2.5 \alpha^{0.5}} \\
& \text { B level of service }(\mathrm{CI}=0.5 \sim 1.0) \\
& \beta=0.65+2.3 \cdot e^{-2 \alpha^{0.5}}
\end{aligned}
$$


C level of service $(\mathrm{CI}=1.0 \sim 5.0)$

$\beta=0.40+2.3 \cdot e^{-1.8 \alpha^{0.5}}$

Likewise, other evaluation indexes can also apply this method to reach the traffic density and the facilities' initial number $\alpha-\beta$ relation curve, as shown in Figures 4 to 6 .

\section{Results of $M / M / N$ queuing system}

According to table 1 and the results of equations (51)-(53), the relation between the traffic density $(\alpha)$ of each evaluation index and the parameter of the initial number of facilities $(\beta)$ can be inducted into the general equation (54), which this study refers to as the " $\alpha-\beta$ relation equation". The variations of each parameter, for each system's evaluation indexes under diverse

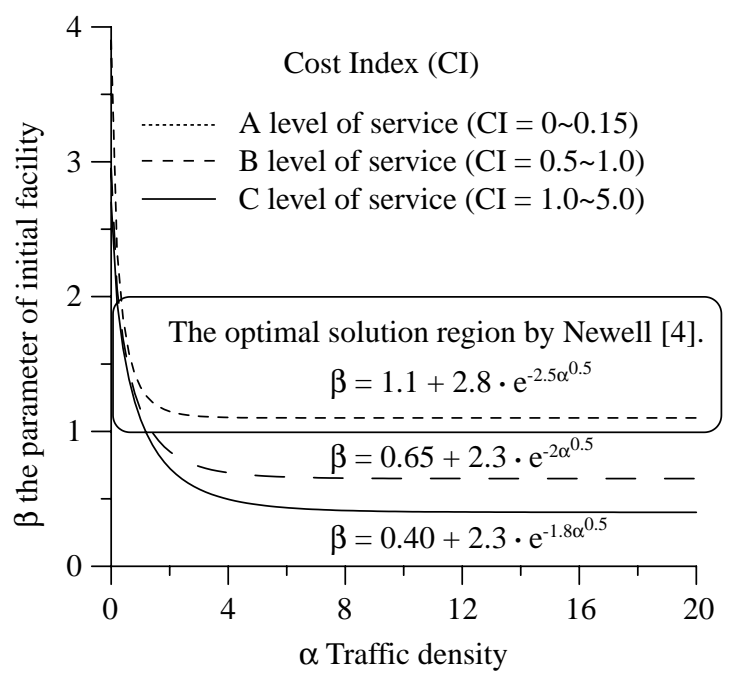

Fig. 3. Relationship between $\alpha$ and $\beta$ at different levels of service (CI).

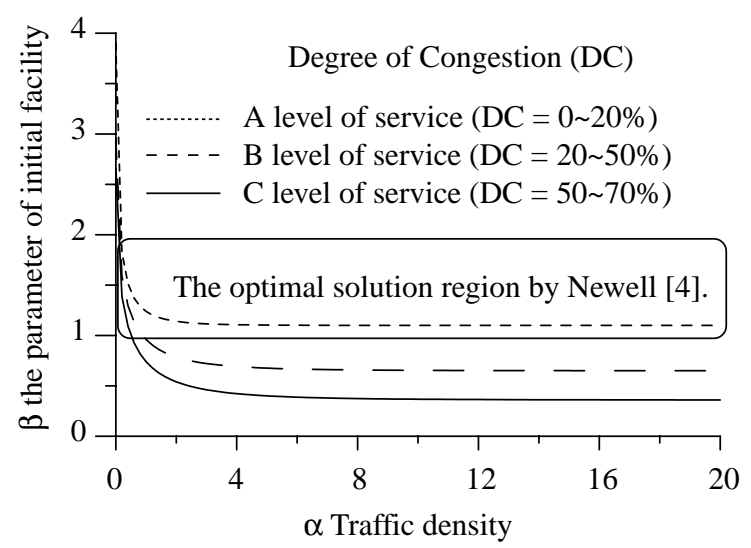

Fig. 4. Relationship between $\alpha$ and $\beta$ at different levels of service (DC). levels of service, are presented in table 2.

$$
\beta=\boldsymbol{a}+\boldsymbol{b} \cdot e^{-\boldsymbol{c} \alpha^{d}}
$$

According to table 2, the system's number of initial facilities can vary a great deal, depending on the different levels of traffic service and the diverse evaluation indexes. If we take DC as an example, when traffic service is at level $\mathrm{A}$, the initial number is measured by the equation $[\alpha+(1.1 \sim 2.8) \sqrt{\alpha}]$; when service is at level $\mathrm{B}$, the initial number is calculated by the equation $\left[\alpha+(0.65 \sim 2.3)_{\sqrt{\alpha}}\right]$; and when service is at level $\mathrm{C}$, the initial number is calculated by the equation $\left[\alpha+(0.37 \sim 2.3)_{\sqrt{\alpha}}\right]$. When traffic density $\alpha$ is under $0.5, \beta]$ is between 1.1 and 2.8 . The calculated results of other types of the port system evaluation indexes, under diverse levels of service, are explained as follows:

1. Parameter "a" is closely associated with level of service. When the level of service was at level A, the parameter a of each index was 1.1 and 1.5 , respectively. When the level of service was at level $\mathrm{B}$, the parameters were 0.6 and 0.65 , respectively. Moreover, when the

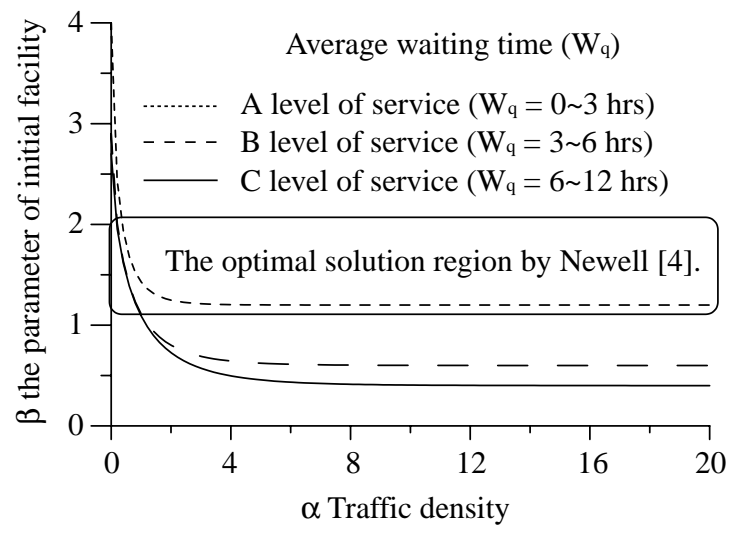

Fig. 5. Relationship between $\alpha$ and $\beta$ at different levels of service $\left(W_{q}\right)$.

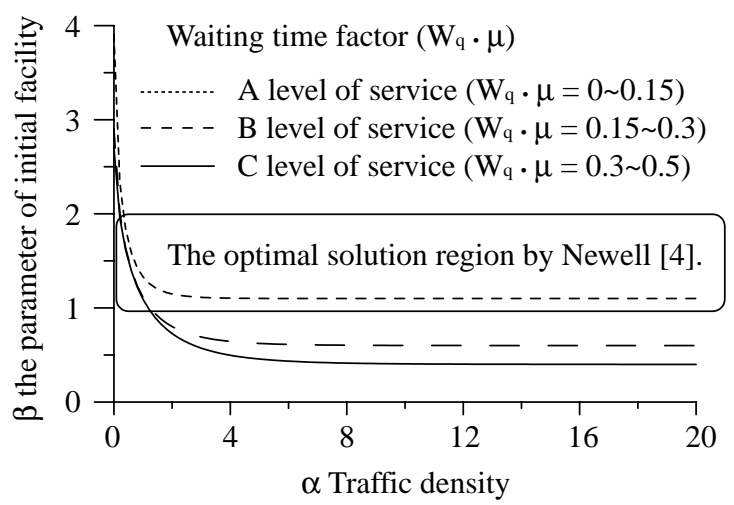

Fig. 6. Relationship between $\alpha$ and $\beta$ at different levels of service ( $W_{q}$ - $\mu)$. 
level of service was at level $\mathrm{C}$, the parameters were 0.37 and 0.4 , respectively. Parameter " $a$ " of the DC index was between 0.37 and 1.1 ; the parameters of $W_{q}$ indexes and $W_{q} \cdot \mu$ indexes were both $0.4-1.5$, while the parameters of the CI indexes were between 0.4 and 1.1. This result shows that when DC and CI were used as evaluation indexes, in terms of the variation of the level of service, the parameters were less varied.

2. Parameter " $b$ " was less apparent than parameter $a$, in relation to level of service. When service was at level $\mathrm{A}$, the " $b$ " parameters in each index were all 2.8. Regarding the $\mathrm{B}$ or $\mathrm{C}$ levels of service, the parameters were both 2.3.

3. Parameter " $c$ " was more apparent than parameter $b$ in relation to level of service. When the level of service was at level A, except for the parameter of the DC index (which is 3), parameter " $b$ " of all other indexes was 2.5. Regarding the $\mathrm{B}$ and $\mathrm{C}$ levels of service, the parameters were 2 and 1.8 , respectively.

4. Parameter " $d$ " varied with the evaluation index and was not influenced by the level of service. Regarding the DC or CI index, their parameters were both 0.5 , while the parameters of $W_{q}$ and $W_{q} \cdot \mu$ were 0.7 .

Judging from the above analysis, we can see that the cost function $\mathrm{CI}$ index varied the least, with changes in service level, while the degree of variation of the DC index was higher than that of the CI index. Concerning the $W_{q}$ and $W_{q} \cdot \mu$ indexes, their parameters were the same, because of their similar natures.

\section{NUMERICAL EXAMPLES}

This paper has quoted examples from the study cases of Plumlee [8] and Noritake [6]. The basic data

Table 2. Variation of parameters in the $\alpha-\beta$ relation equation

\begin{tabular}{clllll}
\hline $\begin{array}{c}\text { Parameters } \\
\text { L.O.S. Indices }\end{array}$ & $\mathrm{a}$ & $\mathrm{b}$ & $\mathrm{c}$ & $\mathrm{d}$ \\
\hline \multirow{3}{*}{$\mathrm{A}$} & $\mathrm{DC}$ & 1.1 & 2.8 & 3 & 0.5 \\
Level & $W_{q}$ & 1.5 & 2.8 & 2.5 & 0.7 \\
& $W_{q} \cdot \mu$ & 1.5 & 2.8 & 2.5 & 0.7 \\
& $\mathrm{CI}$ & 1.1 & 2.8 & 2.5 & 0.5 \\
\hline \multirow{3}{*}{ B } & $\mathrm{DC}$ & 0.65 & 2.3 & 2 & 0.5 \\
Level & $W_{q}$ & 0.6 & 2.3 & 2 & 0.7 \\
& $W_{q} \cdot \mu$ & 0.6 & 2.3 & 2 & 0.7 \\
& $\mathrm{CI}$ & 0.65 & 2.3 & 2 & 0.5 \\
\hline \multirow{3}{*}{ C } & $\mathrm{DC}$ & 0.37 & 2.3 & 1.8 & 0.5 \\
Level & $W_{q}$ & 0.4 & 2.3 & 1.8 & 0.7 \\
& $W_{q} \cdot \mu$ & 0.4 & 2.3 & 1.8 & 0.7 \\
& $\mathrm{CI}$ & 0.4 & 2.3 & 1.8 & 0.5 \\
\hline
\end{tabular}

for cargo management of a specific port is as follows: annual operation time $T=365$ days; cargo management quantity $Q=1.8 \times 10^{6}$ tons; cargo management efficiency $R=800$ tons per day.

\section{Comparison of methods to determine initial number of berths (regardless of the cost function)}

Table 3 points out the comparison between a system's initial number of facilities, using three different indexes, regardless of the cost, under different service levels, and that of other methods. It shows that the optimal number of facilities for each index should change in accordance with diverse service levels. In contrast, the other methods do not take the differences in the system's service levels into account. Thus, the initial number of facilities do not change with variations in the service levels of the evaluation indexes. Accordingly, their effectiveness and applicability are lower.

(1) This study method was used to measure the initial number of facilities. When DC was $10 \%$, and at service level A, as defined by this study, the parameters in table 2. were applied to equation (54), obtaining $\beta=1.102$, which was later applied to equation (50) $\mathrm{N}_{0}=8.89$. Therefore, we were able to conclude that the initial number of berths was 9 . Likewise, when DC $=15 \%$ and $30 \%$, the initial number of facilities was 9 and 8 , respectively.

(2) When using the method of Plumlee [8] and Noritake [6]:

Table 3. The comparison of three methods showing the initial number of berths with different indexes and levels of service $(\alpha=\lambda / \mu=6.16)$

\begin{tabular}{|c|c|c|c|c|c|c|}
\hline \multirow{2}{*}{\multicolumn{2}{|c|}{ Indexes }} & \multirow{2}{*}{$N^{*}$} & \multirow{2}{*}{$\begin{array}{c}\text { This } \\
\text { study } \\
N_{o}\end{array}$} & \multirow{2}{*}{$\begin{array}{c}\text { Plumlee [8] } \\
\text { and Noritake } \\
{[6] N_{o}}\end{array}$} & \multicolumn{2}{|c|}{ Newell [4] $N_{o}$} \\
\hline & & & & & $\beta=1$ & $\beta=2$ \\
\hline \multirow{3}{*}{ DC } & $10 \%$ & 10 & 9 & 7 & 9 & 12 \\
\hline & $15 \%$ & 9 & 9 & 7 & 9 & 12 \\
\hline & $30 \%$ & 8 & 8 & 7 & 9 & 12 \\
\hline \multirow{3}{*}{$W_{q}$} & $0.5 \mathrm{hrs}$ & 10 & 9 & 7 & 9 & 12 \\
\hline & $4 \mathrm{hrs}$ & 9 & 9 & 7 & 9 & 12 \\
\hline & $5 \mathrm{hrs}$ & 8 & 8 & 7 & 9 & 12 \\
\hline \multirow{3}{*}{$W_{q} \cdot \mu$} & 0.03 & 10 & 9 & 7 & 9 & 12 \\
\hline & 0.1 & 9 & 9 & 7 & 9 & 12 \\
\hline & 0.2 & 8 & 8 & 7 & 9 & 12 \\
\hline
\end{tabular}

a. $\mathrm{N}^{*}$ is optimum number of berths.

b. The shaded parts show that the initial number of facilities already exceeds the optimal number of facilities. 
$S>(Q / R T)=\alpha=\lambda / \mu=6.16$

So the initial number of berths was 7

(3) When using the method of Newell [4]:

Traffic density $\alpha=\lambda / \mu=Q / R T=6.16$

When $\beta=1, N_{0}=[\lambda / \mu+(1) \sqrt{(\lambda / \mu)}]=8.64$

So the initial number of berths was 9

When $\beta=2, N_{0}=[\lambda / \mu+(2) \sqrt{(\lambda / \mu)}]=11.12$

The initial number of berths was 12 , exceeding the optimal facility number of 10 . In the same way, the other shaded parts in table 3 show that the resolved initial number of facilities had already exceeded the optimal facility number.

\section{Comparison of methods to determine initial number of berths (based on cost function)}

In consideration of the cost function, we made a comparison with the above two methods, under different levels of service. The results can be seen in table 4, while the calculation method is laid out as follows:

(1) Using our study method to count the initial number of berths, when CI was 0.25 , we applied the parameters in table 2 to equation (54), gaining $\beta=1.104$, which was later applied to equation (50) $N_{0}=8.89$. Therefore, we concluded that the initial number of berths was 9 . Likewise, when CI $=1.0$ or 5 , the initial number of facilities was 8 and 7 , respectively.

(2) As shown in table 4 , when $\mathrm{CI}=0.25$, the optimal number of facilities was 10 berths; the initial number of facilities derived by this study was 9 berths. In comparison, Noritake [6]'s method resulted in 7 berths. In Newell's [4] method, when $\beta=1$, the results was 9 berths; when $\beta=2$, it was 12 berths.

Table 4. Comparison of different methods of calculating the initial numbers using cost index under different levels of service $(\alpha=\lambda / \mu=6.16)$

\begin{tabular}{|c|c|c|c|c|c|}
\hline \multirow{2}{*}{$\begin{array}{l}\text { Cost } \\
\text { Index } \\
(\mathrm{CI})\end{array}$} & \multirow{2}{*}{$\begin{array}{c}\text { Optimal } \\
\text { number of } \\
\text { berths }\left(\mathrm{N}^{*}\right)\end{array}$} & \multirow{2}{*}{$\begin{array}{l}\text { This } \\
\text { study } \\
\left(\mathrm{N}_{\mathrm{o}}\right)\end{array}$} & \multirow{2}{*}{$\begin{array}{c}\text { Noritake } \\
{[6]} \\
\left(\mathrm{N}_{\mathrm{o}}\right)\end{array}$} & \multicolumn{2}{|c|}{ Newell [4] $\left(\mathrm{N}_{\mathrm{o}}\right)$} \\
\hline & & & & $\beta=1$ & $\beta=2$ \\
\hline 0.25 & 10 & 9 & & & \\
\hline 0.5 & 9 & 9 & & & \\
\hline 1.0 & 8 & 8 & $7 * *$ & $9 * *$ & $12 * *$ \\
\hline 2.5 & 8 & 7 & & & \\
\hline 5.0 & 7 & 7 & & & \\
\hline
\end{tabular}

** regardless of the influence of the cost function, the initial number was fixed.
These results show that our solution was closer to the optimal number of berths.

(3) When CI $=1.0$, the initial number calculated by this study was 8 berths, which is the optimal number. In Newell's [4] method, neither of the solutions exceeded the optimal number of facilities, whether $\beta$ $=1$ or $\beta=2$.

(4) When CI $=5.0$, the initial number reached by this study and by Noritake's [6] method was exactly the optimal number of facilities: 7 berths. In Newell's [4] method, neither of the solutions exceeded the optimal number of facilities, whether $\beta=1$ or $\beta=2$.

\section{CONCLUSION}

Our conclusions and suggestions, based on the study results, are stated below:

1. This study found that the initial number of facilities in a queuing system could be determined by the mathematical equation $N_{0}=\alpha+\beta \cdot \sqrt{\alpha}$, in which "the parameter $(\beta)$ of the system's initial number of facilities" will change with different evaluation indexes and diverse levels of service. The Newell [4] method was an exception to this and was included.

2. According to the study results, the relation between traffic density and the parameters of the initial number can be shown by the equation $\beta=a+b \cdot e^{-c \alpha^{d}}$. Based on the analysis of case studies, the solving efficiency and accuracy of the initial number of facilities, reached by this research method, was better than that reached by other methods.

3. This study takes into consideration many different queuing system evaluation indexes, including the degree of congestion, average waiting time, average waiting factors, cost functions, etc. In this way, we were able to determine the system's initial number of facilities. In comparison with other indexes, regardless of cost functions, Cost Index (CI) was more stable. That is, its degree of variation was smaller.

4. A queuing system's initial number of facilities was found to be closely associated with the variation in the different evaluation indexes, and was related to the levels of service shown by these evaluation indexes. Therefore, the relation between the facility's size and its evaluation indexes can be employed to establish a relationship among the different kinds of evaluation indexes.

5. In practice, this study, estimating a queuing system's initial number of facilities, may be applied to: the entrances and exits of public parking lots; the cargo flow centers and gas stations; the cash-out counters of supermarkets or large stores; the wickets at train stations, MRT stations, movie theaters, etc., by estimating of the number of service outlets. In particular, 
when the required facility numbers are increasing, much time can be saved by employing the methods outlined in this study: first by calculating the initial number of facilities and then by obtaining the optimal number of service facilities.

6. In addition, because this study only takes the initial number of facilities of the $M / M / N$ queuing system into account, in future, we may undertake the calculation of the initial number of facilities for other queuing systems such as $M / E_{k} / N, M / G / N, M / M^{x} / N$ and $E_{k} / E_{k} / N$, in order to enhance its applicability.

\section{ACKNOWLEDGEMENTS}

The authors would like to thank the National Science Council of the Republic of China for its support of the research NSC 89-2213-E-019-022. The content of this paper is part of the results from that study.

\section{REFERENCES}

1. Huang, W.C., "The Study of the Characteristics on the Optimal Analysis of Port System," J. Transp. Plan., Vol. 19, No. 2, pp. 241-268 (1990).

2. Huang, W.C., Chishaki, T., and Li, G., "A Study on the
Container Port Planning by Using Cost Function with IND Based on $\mathrm{M} / \mathrm{E}_{\mathrm{K}} / \mathrm{N}$ System," J. East. Asia Soc. Transp. Stud., Vol. 1, No. 1, pp. 263-276 (1995).

3. Lan, L.W. and Teng, J.Y., "Applications of the M/M/S Queuing System to Determine the Optimal Number of Berths," J. Chin. Ins. Civil Hydraul. Eng., Vol. 2, No.4, pp. 369-375 (1990).

4. Newell, G.F., Applications of Queuing Theory, Chapman and Hall, New York (1982).

5. Nicolau, S.N., "Berth Planning by Evaluation of Congestion and Cost," J. Waterw. Harb. Div.- ASCE, Vol. 93, No. 4, pp. 107-132 (1967).

6. Noritake, M., "A Study on Optimum Number of Berths in Public Wharf," Proceedings of the Japanese Society of Civil Engineers, Vol. 278, pp. 113-122 (1978).

7. Noritake, M. and Kimura, S., "Optimum Number and Capacity of Seaport Berths," J. Waterw. Port C.-ASCE, Vol. 109, No. 3, pp. 323-329 (1983).

8. Plumlee, C.H., "Optimum Size Seaport," J. Waterw. Harb. Div.-ASCE, Vol. 92, No. 3, pp. 1-24 (1996).

9. Wanhill, S.R.C., "Further Analysis of Optimum Size Seaport," J. Waterw. Port C.-ASCE, Vol.100, No.4, pp. 377-383 (1974). 